\title{
Cheetah-cub-S: Steering of a Quadruped Robot using Trunk Motion
}

\author{
K. Weinmeister ${ }^{1}$, P. Eckert ${ }^{2}$, H. Witte ${ }^{1}$ and A.-J. Ijspeert ${ }^{2}$
}

\section{Motivation And State of The ART}

In 2013, Sproewitz and co-workers [1] introduced the compliant quadruped robot Cheetah-cub. It consisted of four legs which were actuated by two motors each and open loop controlled via a network of central pattern generators (CPG). The main feature of Cheetah-Cub was its leg design, an advanced spring loaded panthograph leg (ASLP-leg) [2], following the model of small quadrupedal mammals [3]. Due to its passive compliant properties, effects like selfstabilization during open loop locomotion could be observed. To the author's best knowledge, the robot achieved with its approx. $1.42 \mathrm{~m} / \mathrm{s}$ (6.9 body lengths and / Froude number of 1.3) the fastest speed for legged robots under $30 \mathrm{~kg}$ and over $0.3 \mathrm{~kg}$ (i.e. above insect scale). However, one feature, present in almost all terrestrial animals, that was missing is the ability to steer. Changing directions is after forward locomotion the most important feature to achieve higher maneuverability and thus increases the set of possible applications. The simple possibility to steer by strong modulation of the gait can lead to low dynamics and unsatisfying movements. Therefore, inspired by properties of mammalianlike locomotion advantageous for robot motion [4], we set up the following hypothesis: The usage of an actuated spine will significantly improve the steering ability of a quadruped robot compared to the use of a rigid spine combined with parameter changes in the legs' control.

\section{A. Vertical bending spines and turning via foot-placement}

In the past, different robots with a spine-like structure were introduced. In 1996, a planar quadruped robot of the MIT used an articulated spine during bounding to improve its locomotion [5] resulting in augmented thrust. In contrast, a fully passive and compliant mobile platform was developed by Kani in 2011. Three different spine types were tested and the robot Fanari was able to gallop down a slope just using gravity [6]. Different spine designs were also investigated by Eckert in 2014 in the modular, compliant quadruped Lynx [7]. Again, the role of the spine during bounding was investigated. A parallel actuated compliant spine was implemented into the "open loop quadruped" Canid to analyse the role of the spine during bounding and galloping [8]. Due to open loop, touchdowns were uncoordinated and no useable bounding gait could be achieved. To sum up, all these spines were designed to improve forward locomotion while only vertical bending was allowed. Therefore, turning has to be done based on other ideas, e.g. by modulating individual foot placement. Matos and Santos applied a CPG to a SONY Aibo to make omnidirectional locomotion possible by controlling each leg individually [9]. Ajallooeian developed a controller for a cat-sized quadruped robot and made turning motion possible by using ab-/adduction at the hip [10]. WildCat is another quadruped which uses ab-/adduction to

\footnotetext{
${ }^{1}$ Chair of Biomechatronics, Faculty of Mechanical Engineering, Technische Universit"at Ilmenau, Germany http: //www.tu-ilmenau.de/biomechatronik/

${ }^{2}$ Biorobotics Laboratory, Ecole Polytechnique Federale de Lausanne, Switzerland http: / / biorob.epfl.ch/
}

change direction [11]. Tekken4 has four DoF at each leg and changes direction by adapting their yaw angles[12]. Changing directions with a rigid trunk is thus possible but requires an overall high DoF of the legs and thus more complex control approaches.

\section{B. Lateral bending spines}

Zhao developed a multi-segment, biologically inspired and compliant spine for a quadruped robot. Due to a sine wave motion of the spine and an asymmetrical friction material at the feet, the robot was able to move forward and turn through stronger lateral spine bending. The robot achieved an average angular speed up to $6.2 \%$ [13]. This approach seems to be very promising but requires complex parts and does not move very dynamically. Furthermore, we look at robots based on amphibians and reptiles as for them lateral bending of the spine in a complex manner is crucial. Pleurobot [14] and Salamandra robotica II [15] are two bio-inspired salamanderlike robots for ground and water locomotion. The former has 27 and the latter 12 actuated DoFs and both show good turning behavior. Instead of increasing the overall complexity by increased DoF and more complex control algorithms as dicussed before, we aimed for keeping the control structure as simple as possible while implementing intelligent bioinspired mechanics. The approach of a simplified lateral bending compliant spine from amphibians in combination with Cheetah-cub's test-proven ASLP-legs serves a promising basis for a new mobile platform: Cheetah-cub-S.

\section{APPROACH}

\section{A. Mechanical concept}

Fig. 1a shows the setup of the spine at neutral position. The lateral deflection of the spine can be determined actively by the motor placed in between the fore an hind trunk segment while external loads are absorbed by the compliant element. Leaf springs (Green line) were attached to each side of the motor. The revolute joint and the compliant element with bending over a wide range are thus implemented at once. Fig. 1b shows the bending of both leaf springs induced by shorten- and lengthening of the cables (Dashed lines).

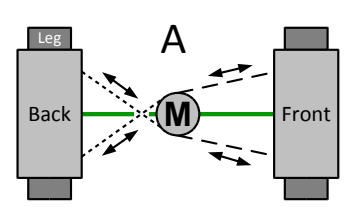

(a)

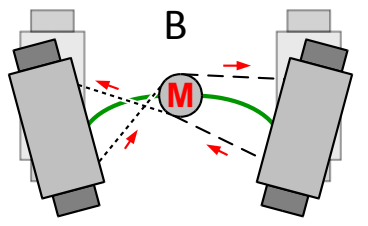

(b)
Fig. 1: M - Motor (black = neutral, red = powered), Dashed line - Cable mechanism, Arrows - Movement of cables, Green line - leaf spring; A: General setup at neutral position, B: Motor turns clockwise to initialize curved path

\section{B. Spine segment - implementation and range of motion}

Two leaf springs on the top connect the motor with both trunk modules. They are dimensioned to avoid bending due 
to gravity and thus, to allow lateral bending only. Furthermore, to decrease torsion due to external loads without loosing the ability of lateral bending, one identical leafspring is added in the bottom. The centered motor was directly connected to the springs. The cables favored in the concept were exchanged by a back-drivable bar mechanism in the final version to enable the deflection and ensure additional rigidity of the spine against torsion. The cabledriven prototype was generally still too flexible. Fig. 3 shows

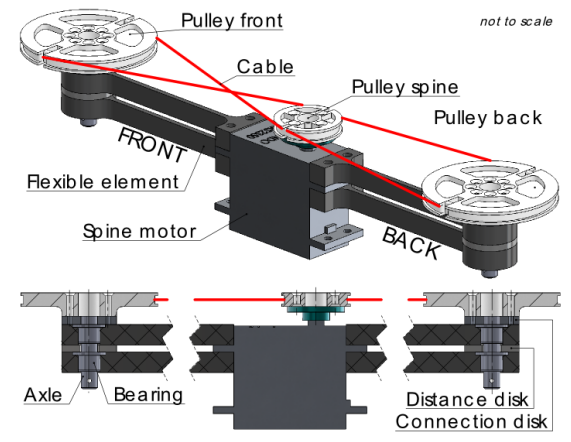

Fig. 2: Spine design: Isometric and section view - clarified CAD model (without screws, etc.)

the ranges of motion of spine, hip and knee. Each spine segment bends to $\pm 5^{\circ}$ which corresponds to a total spine deflection of $\pm 10^{\circ}$. The ASLP-legs perform an oscillatory movement as seen in Cheetah-Cub.

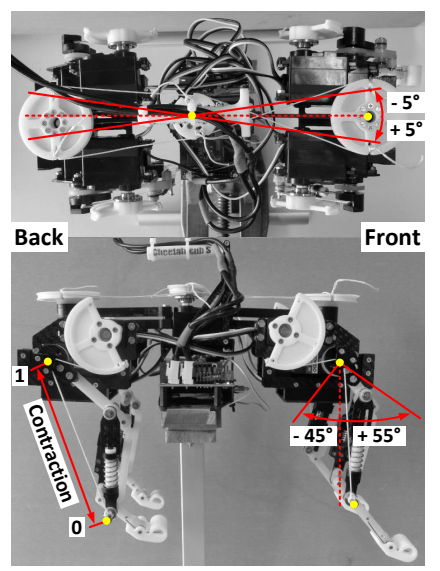

Fig. 3: "head" of the robot to the right; Range of motion: Top - Spine: $\pm 10^{\circ}$; Bottom right - Hip: $+55^{\circ} / 45^{\circ}$ (Front legs)and $\pm 45^{\circ}$ (Hind legs); Bottom left - Knee: Full (1) or no (0) contraction of the diagonal spring; Reference points: hip/spine motor and ankle/trunk joint (yellow)

\section{Experiments}

To ensure uniformity, the spine deflection was divided into steps of $2^{\circ}$, i.e. from $-10^{\circ}$ to $10^{\circ}$. Ten attempts of trotting with a minimum of two complete circles were recorded with a motion capturing system for each step. If the turning radius exceeded the test area, the movement was recorded as far as possible. Furthermore, attempts were marked as not successful if the robot fell over (Fig. 4).

\section{Result and Analysis}

The robot achieved its sharpest turn with a $10^{\circ}$ spine deflection at a minimum radius of $0.51+/-0.07 \mathrm{~m}$ at $0.31 \mathrm{~m} / \mathrm{s}$. $97 \%$ of all 110 attempts were successful with Cheetah-cub-S falling only when unintentionally hitting the

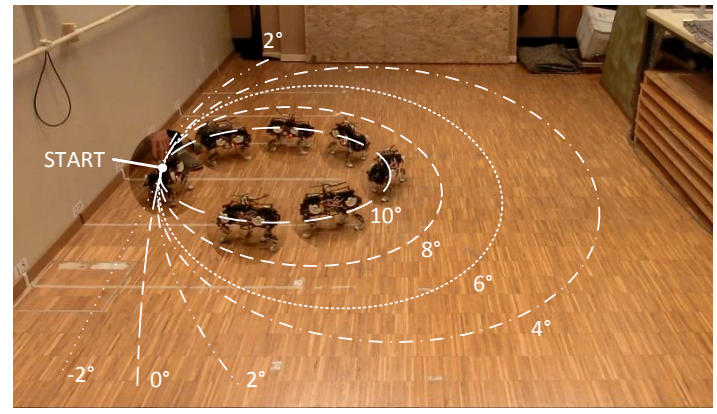

Fig. 4: Simplified trajectory of different spine deflections: Full turning up to $4^{\circ}$, negative spine deflections were left out for clarity reasons

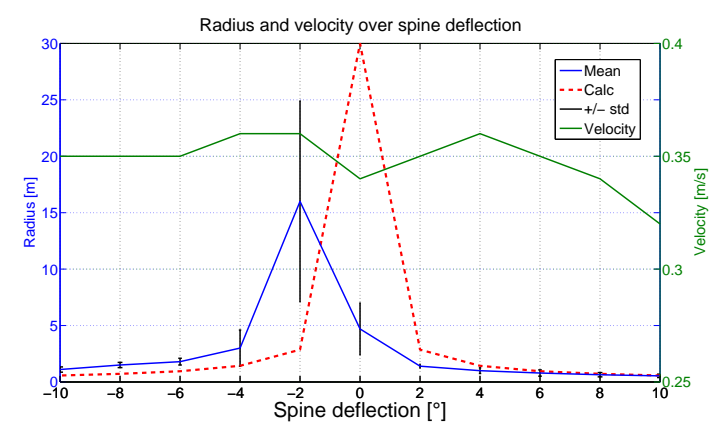

Fig. 5: Results of experiments: Radius and velocity over spine deflection: Calc - calculated radii (with Single-TrackModel, $30 \mathrm{~m}$ at $0^{\circ}$ represents $\infty$ ), std - standard deviation

wall during the run. An asymmetry exists by comparing experimental and calculated radii. It is caused by a backlash of the bar mechanism and material fatigue due to extensive testing. Therefore, the spine experienced a pre bending towards one side. A comparable (offsetted) radius can be observed. The velocity did not change significantly during the experiments and reached its maximum of $0.36 \mathrm{~m} / \mathrm{s}$ at straight locomotion, a strong difference to Cheetah-Cub. More experiments are needed to find better gait-parameters and hopefully reach higher forward speeds with good gaitcharacteristics. Low friction of the feet in combination with wooden test underground caused undesired sliding motion which increased with greater spine deflection due to additional dynamic forces. To compare the steering ability of Cheetah-Cub and Cheetah-Cub-S, new control-parameters were calculated and implemented in the former. As observed in cars during curved path driving, the amplitude of the inner leg (wheel) had to be approximately $20 \%$ less than the outer to achieve the same turning radius $(0.5 \mathrm{~m})$. No changes in direction occurred. One possible reason was the high locomotion-frequency which made it impossible for the motors to reach the commanded amplitudes. The frequency was not lowered to keep the comparison between the two robots at a more dynamic speed. The amplitudes were thus hand-tuned until a ratio of $5^{\circ}$ (inside) to $50^{\circ}$ (outside). The full circle was achieved after $20 \mathrm{~s}$ which correlates to $\approx 0.16 \mathrm{~m} / \mathrm{s}$. Therefore, the velocity was half the one of Cheetah-cub-S and the gait changed from a trot to a full contact sliding gait. Hence, the robot was prone to changes of the ground and unstable. Moreover, the differences in amplitude of calculation and reality were disproportional. This is due to the origin of the model in automotive application. The approximated amplitude-ratio corresponded to a very long distance traveled during stance-phase but in reality the foot 
touched the ground less. To achieve the desired ground contact, the foot trajectory has to be controlled and adjusted during locomotion, which is not a trivial task to solve.

\section{CONCLUSION AND Discussion OUTLINE}

With the help of the artificial spine, a sharp turning was obtained and the turning radius was reduced to $0.51 \mathrm{~m}$ at $0.31 \mathrm{~m} / \mathrm{s}$. Furthermore, the design allowed a human operator (or a higher-level navigation controller) to modulate the spine deflection and therefore to steer the robot in its environment (Fig. 6).

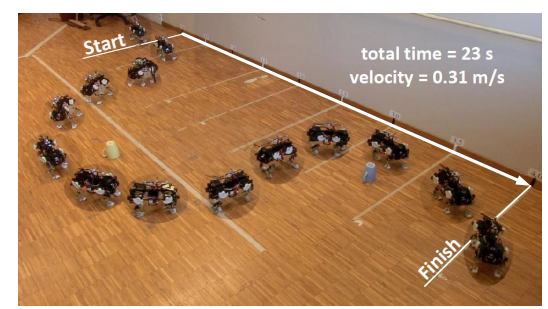

Fig. 6: Slalom - Experiment: Recorded movement of Cheetah-cub-S represented by snapshots (without cable), white arrow represents $4 \mathrm{~m}$ distance

In comparison to its role model, Cheetah-Cub, the dimensions are similar apart from the mass, forward and turning velocity. Although the additional weight of $60 \mathrm{~g}$, caused by the spine actuator, Cheetah-Cub-S is able to almost turn twice as fast as Cheetah-Cub and keep the characteristics of a normal trotting gait. The forward speed decreased drastically, caused by the non-optimized gait and much lower supply voltage than Cheetah-Cub (9V instead of 14V). All in all, Cheetahcub-S introduces a novel, modular approach to enable steering via trunk motion without the consideration of individual foot placement or gait specialization and thus little increase in control complexity. Furthermore, we implemented only one additional DoF but increased the maneuverability (also real time by one simple control input) markedly even though the locomotion-parameters can and will still be optimized (confirming the hypothesis). Therefore, the overall design showed its advantages which makes Cheetah-cub-S a very successful robot in turning via spine deflection. Questions and topics we hope to find input from the AMAM2015 community:

- Usage of a modular concept (cp. [16])

The system was based on modularity which makes it possible to replace parts by others. How does the performance change if different legs, spine segments (front and back) or motors will be used? What are interesting modifications that could be implemented? Should an abb-/adduction implemented and if yes is it worth the higher complexity?

- Foot trajectory and gait

Due to open loop control, the trajectory of the feet was not directly controlled. Can the performance be improved by closing the loop and controlling every foot individually? What sensors would be needed? The ground had a great effect during the experiments. How can friction be used beneficiary e.g. by a special foot design?

- Spine and straight locomotion

Additionally to using the spine for turning, it could also be used during straight locomotion and varied within one stepping cycle. This approach can be seen in amphibians and reptiles, i.e. salamanders. Is this worth exploring or does it stray too much from a mammal like approach? What could be the benefit of this? Do other quadrupedal gaits make excessive use of a lateral bending spine and how?

- Experimental platform

Cheetah-cub-S offers the opportunity to add additional control layers for navigation and obstacle avoidance. Observation of the environment and trajectory planning can be done independently and modulate the locomotion by changing the steering commands of the spine. What could be the preferred principle for sensors and algorithms, that don't exert too high complexity and thus comply with the "as simple as possible, as complex as needed" approach of the Cheetah-Cub-family.

\section{Presentation and Travel Grant}

We would like to present in a short talk or short hardware demo. No travel grant is needed.

\section{REFERENCES}

[1] A. Spröwitz, A. Tuleu, M. Vespignani, M. Ajallooeian, E. Badri, and A. J. Ijspeert, "Towards dynamic trot gait locomotion: Design, control, and experiments with Cheetah-cub, a compliant quadruped robot," The International Journal of Robotics Research, vol. 32, pp. 932-950, 2013.

[2] H. Witte, R. Hackert, W. Ilg, J. Biltzinger, N. Schilling, F. Biedermann, M. Jergas, H. Preuschoft, and M. S. Fischer, "Quadrupedal Mammals as Paragons for Walking Machines," in Proceedings of Adaptive Motion in Animals and Machines, 2000, p. 6 pp.

[3] H. Fischer, M.S.; Witte, "The functional morphology of the threesegmented limb of mammals and its specialities in small and mediumsized mammals." Proc. Europ. Mechanics Coll. Euromech 375 Biology and Technology of Walking: 10-17., 1998.

[4] H. Witte, M. S. Fischer, N. Schilling, W. Ilg, R. Dillmann, M. Eckert, and J. Wittenburg, "Konstruktion vierbeiniger Laufmaschinen," Konstruktion, vol. 52, no. 9, pp. 46-50, 2000.

[5] K. F. Leeser and M. Raibert, "Locomotion Experiments on a Planar Quadruped Robot with Articulated Spine," Mechanical Engineering, 1996.

[6] M. H. H. Kani, M. Derafshian, H. J. Bidgoly, and M. N. Ahmadabadi, "Effect of flexible spine on stability of a passive quadruped robot: Experimental results," in 2011 IEEE International Conference on Robotics and Biomimetics, ROBIO 2011, 2011, pp. 2793-2798.

[7] P. Eckert, A. Sproewitz, H. Witte, and A. J. Ijspeert, "Comparing the effect of different spine and leg designs for a small, bounding quadruped robot," in Dynamic Walking, 2014.

[8] J. L. Pusey, J. M. Duperret, G. C. Haynes, R. Knopf, and D. E. Koditschek, "Free-standing leaping experiments with a powerautonomous elastic-spined quadruped," SPIE Defense, Security, and Sensing, vol. 8741, p. 87410W, 2013.

[9] V. Matos and C. P. Santos, "Omnidirectional locomotion in a quadruped robot: A CPG-based approach," in IEEE/RSJ 2010 International Conference on Intelligent Robots and Systems, IROS 2010 Conference Proceedings, 2010, pp. 3392-3397.

[10] M. Ajallooeian, S. Pouya, A. Sproewitz, and A. J. Ijspeert, "Central Pattern Generators augmented with virtual model control for quadruped rough terrain locomotion," in Proceedings - IEEE International Conference on Robotics and Automation, 2013, pp. 3321-3328.

[11] M. Raibert, K. Blankespoor, G. Nelson, R. Playter, and Others, "Bigdog, the rough-terrain quadruped robot," in Proceedings of the 17th World Congress The International Federation of Automatic Control Seoul, Korea, 2008, pp. 10823-10825.

[12] H. Kimura, Y. Fukuoka, and H. Katabuti, "Mechanical Design of a Quadruped“Tekken3\&4”and Navigation System Using Laser Range Sensor," in INTERNATIONAL SYMPOSIUM ON ROBOTICS, vol. 36. unknown, 2005, p. 10.

[13] Q. Zhao, H. Sumioka, and R. Pfeifer, "The Effect of Morphology on the Spinal Engine Driven Locomotion in a Quadruped Robot," in The 5th International Symposium on Adaptive Motion of Animals and Machines (AMAM2011), 2011, pp. 51-52.

[14] "Pleurobot." [Online]. Available: http://biorob.epfl.ch/pleurobot

[15] A. Crespi, K. Karakasiliotis, A. Guignard, and A. J. Ijspeert, "Salamandra Robotica II: An amphibious robot to study salamander-like swimming and walking gaits," IEEE Transactions on Robotics, vol. 29, pp. 308-320, 2013.

[16] R. Eisold, W. Kempf, C. Schilling, and H. Witte, "A Modular Concept for a Biologically Inspired," in In Robot Motion and Control. Springer London, 2009, pp. 391-400. 